\title{
Developed New Procedure for Low Concentrations of Hydrazine Determination by Spectrophotometry: Hydrazine-Potassium Permanganate System
}

\author{
S. Ganesh ${ }^{1}$, Fahmida Khan ${ }^{2}$, M. K. Ahmed ${ }^{1}$, P. Velavendan ${ }^{1}$, N. K. Pandey ${ }^{1}$, U. Kamachi Mudali ${ }^{1}$ \\ ${ }^{1}$ Reprocessing Group, Indira Gandhi Centre for Atomic Research, Kalpakkam, India; ${ }^{2}$ Department of Chemistry, National Institute of \\ Technology, Raipur, India. \\ Email: fkhan.chy@nitrr.ac.in
}

Received October $12^{\text {th }}, 2011$; revised November $23^{\text {rd }}, 2011$; accepted January $9^{\text {th }}, 2012$

\begin{abstract}
An indirect, sensitive and accurate method for the determination of trace amounts of hydrazine is described. In this proposed the spectrophotometric method is based on its reduction properties of hydrazine with a known concentration of potassium permanganate to reduce the colour. The absorbance of unreduced permanganate is measured the colour difference at different wavelengths 546 and $526 \mathrm{~nm}$ which show an absorption spectrum with hydrazine. Hydrazine can be determined in the range of $100-700 \mu \mathrm{g} / \mathrm{ml}$ with correlation coefficient of 0.999 and relative standard deviation $1 \%$. The method is successfully applied for the determination of hydrazine in water streams in nuclear reactors/purex process/boiler water and polluted water samples.
\end{abstract}

Keywords: Hydrazine/Potassium Permanganate; UV-Vis Spectrophotometry; Reducing Property; Water Streams; Nuclear Reactors

\section{Introduction}

Hydrazine and its analogues have found various applications in many industrial, agriculture and other fields, including the manufacture of metal films, photographic chemicals, antioxidants, insecticides and blowing agents for plastics. In addition, hydrazines and their derivatives are suspected to be carcinogens. Hydrazine is widely used as a scavenger to remove traces of oxygen in boiler feed water system [1]. Several methods have been described for the determination of trace amounts of hydrazine, including spectrophotometry, gas chromatography [2] and ion chromatography [3]. Most of these are based on the reaction of hydrazine with aromatic aldehydes such as benzaldehyde [4], salicylaldehyde [5], p-dimethylaminobenzaldehyde [6], pentafluorobenzaldehyde [7] and 5-nitro-2-hydroxybenzaldehyde [8] to form aldazines. Accordingly, their determination at the micro level is of great importance. Despite the accuracy of most of the redox methods $[9,10]$ used for the determination of hydrazines, only few are suitable for extremely dilute solutions, and some require carefully controlled conditions. Most of the spectrophotometric procedures available so far in the literatures [11-22] are tedious and involve the use of rare and expensive colour reagents. The present work describes a very simple, sensitive and accurate spectrophotometric procedure for the determination of ppm concentrations of hydrazine salts based on reaction with potassium permanganate and measurement of the decolourisation of permanganate.

\section{Experimental}

\subsection{Instrumentation}

Fiber optic aided spectrophotometery with $1 \mathrm{~cm}$ path length probe is used for measuring absorbance.

Metrohm make modular Ion-chromatograph equipped with 820 IC separation center, lambda 1010 IC detector (UV-VIS), 818 IC pump (Isocratic), 833 IC liquid handling unit and 830 IC interface and a Metrohm Post column derivatization kit were used. Sample was injected through a $20 \mu \mathrm{L}$ PEEK loop fitted with injector. IC-net 2.3 metrohm software was used for instrument control and data acquisition.

\section{Ion Chromatographic Conditions}

Ion chromatograph: Metrohm modular Ion chromatography system

Eluent concentration: $5 \mathrm{mM}$ Hydrochloric acid

Eluent flow rate: $0.4 \mathrm{ml} / \mathrm{min}$

Pressure: $13.5 \mathrm{MPa}$

Detector: Lambda 1010 UV-VIS detector. 
Analytical mode: Isocratic

Injection loop: $20 \mu \mathrm{L}$

Temperature: Ambient $\left(25^{\circ} \mathrm{C}\right)$

Run time: $10 \mathrm{~min}$

\subsection{Reagents}

All the reagents used were of analytical grade and double distilled water was always used. A stock solution of hydrazine nitrate (obtained from Orion Chem.Pvt.Ltd., Mumbai) was prepared freshly by dilution to the appropriate volume with water. Fresh work solutions are prepared daily by dilution to the appropriate volume of $3.3 \times$ $10^{-3} \mathrm{M}$ aqueous solutions. The hydrazine nitrate solutions were standardized by titration with standard potassium iodate solutions $[23,24]$. The standard potassium permanganate solutions (Prolabo, purity $=98 \%$ ) were prepared and analyzed by titration with oxalic acid [25].

\section{Procedure}

Suitable aliquot of hydrazine and permanganate were added into a series of $10 \mathrm{ml}$ volumetric flasks and made up to mark with distilled water. The absorbance changes of the solution were measured from 700 to $400 \mathrm{~nm}$ using fiber optic aided spectrophotometry. Blank also was run in the same manner.

\section{Ion-Chromatography}

In order to compare the results obtained by spectrophotometric analysis with those of Ion chromatographic technique, experiment were conducted for the determination of hydrazine present in aqueous phase by formation of yellow coloured azine complex by post column derivatization of hydrazine with (pDMAB) para-dimethylaminobenzaldehyde and then analyzing by Ion chromatographic technique using UV-Vis detector. Various hydrazine standards of known concentrations $(1-10 \mu \mathrm{g} / \mathrm{ml})$ were prepared by dissolving hydrazine nitrate in Millipore water. $20 \mu \mathrm{L}$ of these solutions directly injected into

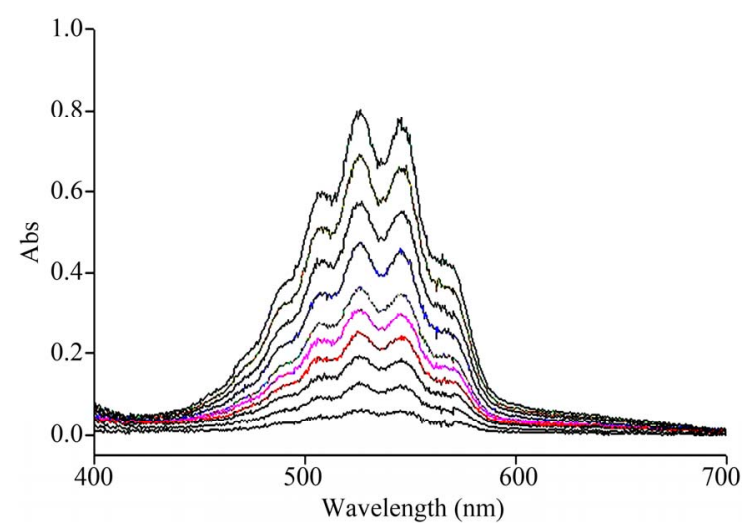

Figure 1. Typical absorption spectrum of $\mathrm{KMnO}_{4}$. the ion-chromatography column connected with UV-Vis detector. Hydrochloric acid $(5 \mathrm{mM})$ with a flow rate of $0.4 \mathrm{ml} / \mathrm{min}$ was used as mobile phase. A mixture of $p$ dimethylaminobenzaldehyde (42 $\mathrm{mM})$, hydrochloric acid $(0.6 \mathrm{M})$ and methanol $(1 \%)$ with a flow rate of $0.6 \mathrm{ml} / \mathrm{min}$ was used as derivatization reagent. A calibration graph was made for the concentration range of hydrazine from 0.05 to $10 \mu \mathrm{g} / \mathrm{ml}$ with RSD $0.807 \%$ and correlation coefficient of 0.9999 . Synthetic aqueous solution containing known concentration of hydrazine was prepared in water, injected $20 \mu \mathrm{L}$ aqueous sample in to Ion chromatography.

\section{Result and Discussion}

In this method determination of hydrazine, the reduction of potassium permanganate with hydrazine has been thoroughly studied and hence, no significant changes were done to adapt the experimental conditions for the determination of hydrazine. The absorption spectrum of potassium permanganate in water shows two absorption maxima at 546 and $526 \mathrm{~nm}$ (Figure 1) for which the molar absorptivities are 2192.18 and $2279.27 \mathrm{~L} \cdot \mathrm{Mol}^{-1} \cdot \mathrm{cm}^{-1}$ respectively. The experimental conditions for the quantitative reduction of potassium permanganate with hydrazine are well established. Similar results were obtained for hydrazine determination with permanganate. The most commonly used p-dimethylaminobenzaldehyde procedures are tedious and suffer from interferences particularly by amines, urea and semicarbazides. High purity and fresh preparation of the colour reagent are essential. Therefore, it was of interest to investigate the possibility of developing a procedure for the determination of hydrazine covering a reasonably wide concentration range and keeping in mind sensitivity, availability and stability of the colour reagent as well as reducing the number of interfering species encountered in the p. dimethylaminobenzaldehyde methods. Standard absorption spectrum and calibration graph for potassium permanganate are shown in Figures 1 and 2. Beer's law was obeyed within

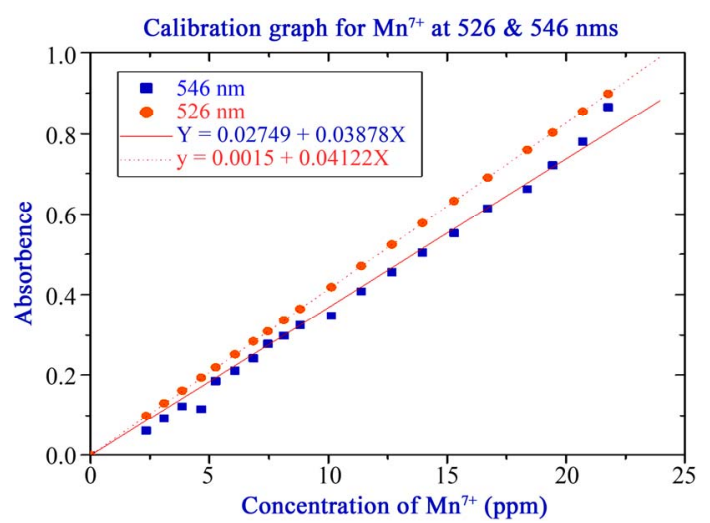

Figure 2. Calibration graph for $\mathrm{KMnO}_{4}$ at $526 \& 546 \mathrm{~nm}$. 
the concentration range investigated. The calculated molar absorptivity of potassium permanganate at 546 and 526 are 2192.18 and $2279.27 \mathrm{~L} \cdot \mathrm{Mol}^{-1} \cdot \mathrm{cm}^{-1}$ respectively. Figure 3 shows that typical absorption spectrum of different concentration of hydrazine with permanganate. Figure 4 shows that calibration graph was made for hydrazine concentration rang of $15-125 \mu \mathrm{g} / \mathrm{ml}$ with correlation coefficient of 0.99549 and RSD 1\% at different wavelengths with potassium permanganate. The accuracy of the present method was checked by determining hydrazine in various samples by both the present method and by an independent technique, namely, Ion chromatography (IC). A typical chromatogram and a calibration curve are shown in Figures 5-6. The results obtained for hydrazine by the proposed and by the ion IC method agreed well within the limits of experimental error and represented in Table 1. Typical results obtained by using this methodology and instrumentation for the determination of hydrazine in water samples are reported in Table 2.

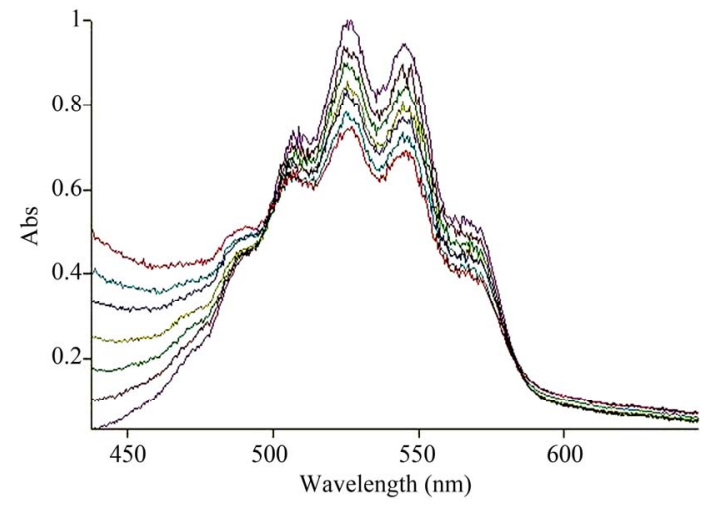

Figure 3. Typical absorption spectrum of hydrazine with $\mathrm{KMnO}_{4}$.

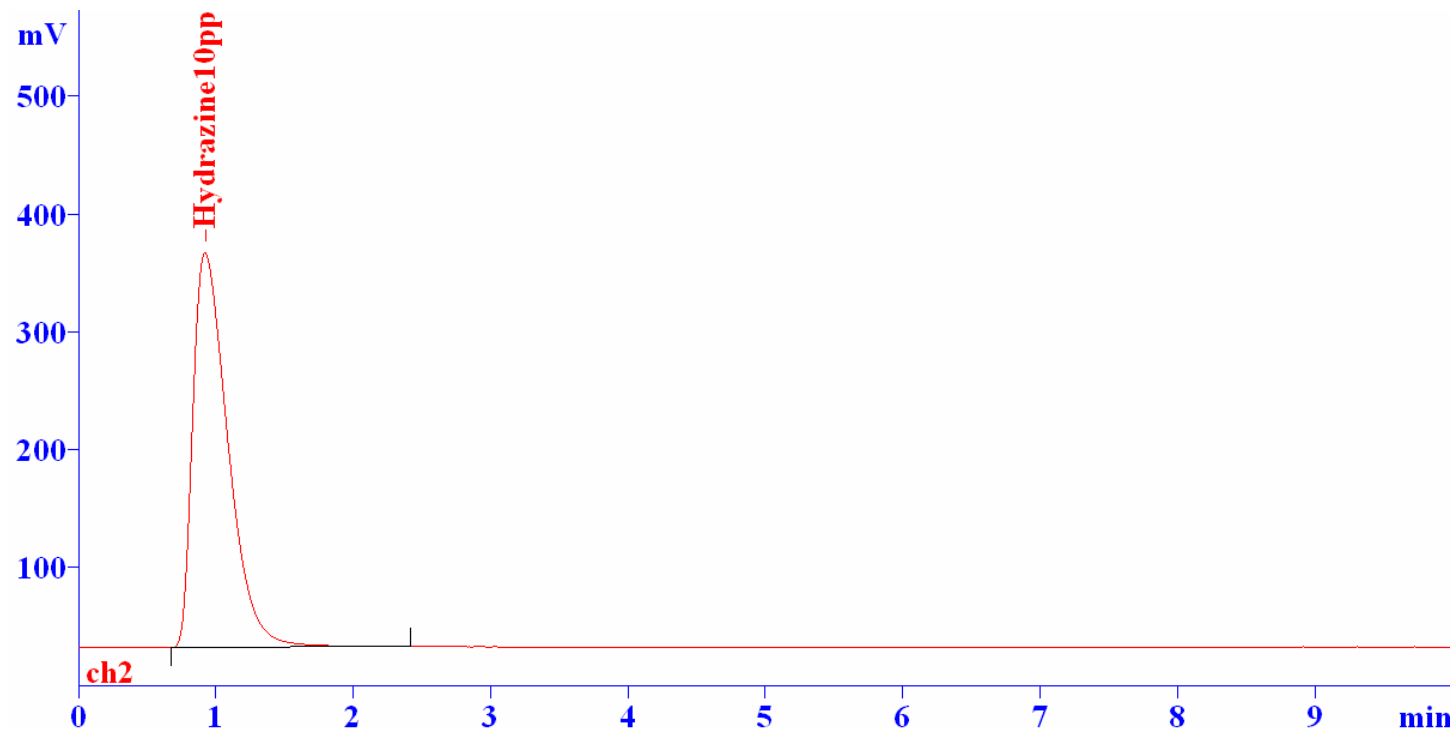

Figure 6. Ion Chromatogram of hydrazine standard.

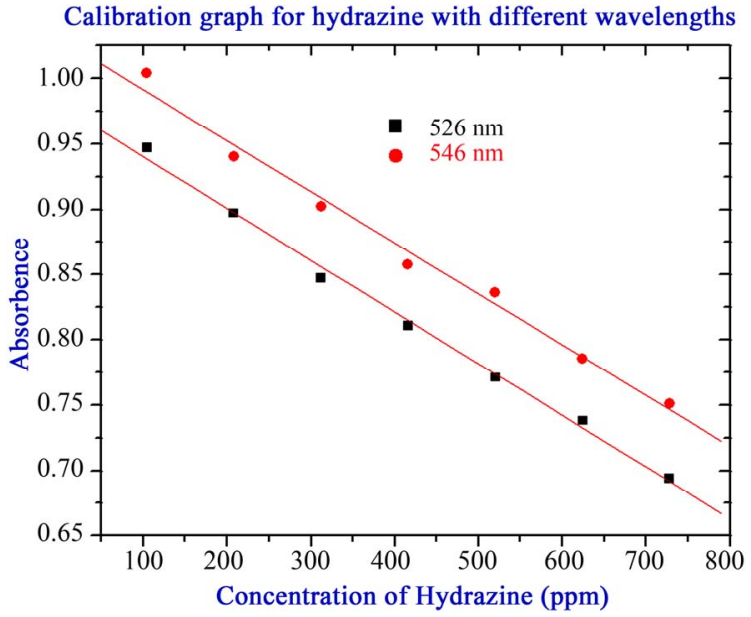

Figure 4. Calibration graph for Hydrazine with potassium permanganate at different wavelengths.

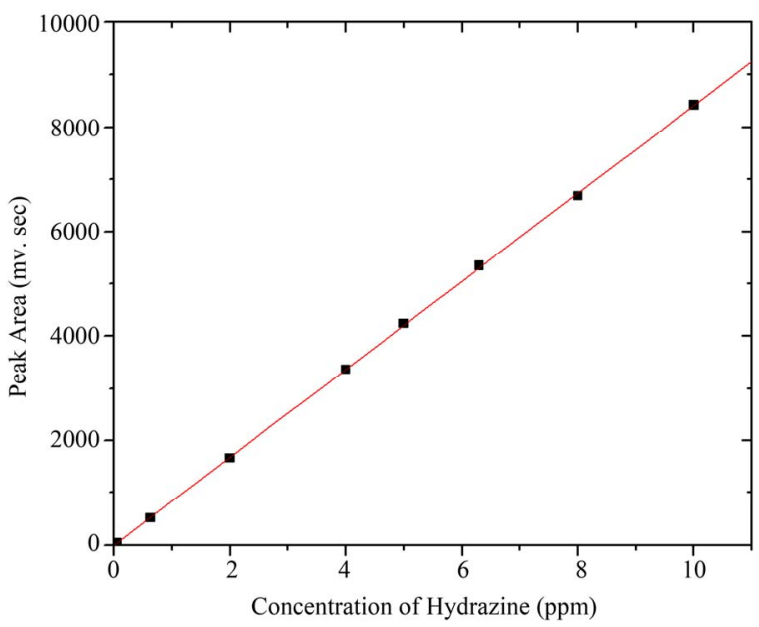

Figure 5. Calibration graph of hydrazine. 
Table 1. Comparsion of two different analytical technique.

\begin{tabular}{ccc}
\hline & \multicolumn{2}{c}{ Concentration of hydrazine $(\mu \mathrm{g} / \mathrm{ml})$} \\
\cline { 2 - 3 } S.No. & Spectrophotometry & $\begin{array}{c}\text { Ion-chromatographic } \\
\text { Technique }\end{array}$ \\
\hline 1 & 6.24 & 6.303 \\
2 & 10.80 & 10.01 \\
3 & 25.30 & 24.80 \\
4 & 12.28 & 12.10 \\
\hline
\end{tabular}

\section{Conclusion}

A simple, rapid and sensitive spectrophotometric procedure is described for ppm determination of hydrazine salts. It is based on reduction of potassium permanganate and measurement of the absorbance at 546 and $526 \mathrm{~nm}$. When compared with other procedures, this method was proved to be of comparable sensitivity and has less interference.

\section{Acknowledgements}

Authors are highly indebted to Shri. S.C.Chetal, Director, IGCAR, Kalpakkam. Authors are grateful to Shri. R. Natarajan, Director, RpG for his valuable suggestions and encouragement during the course of this work.

\section{REFERENCES}

[1] L. F. Audrieth and B. A. Ogg, "The Chemistry of Hydrazines," John Wiley \& Sons, Inc., New York, 1951, p. 225.

[2] V. Vatsala, V. Bansal, D. K. Tuili, M. M. Rai, M. M. Jain, S. P. Srivastava and A. K. Bhatnagar, "Gaschromatographic Determination of Residual Hydrazine and Morpholine in Boiler Feed Water and Steam Condensates," Chromatographia, Vol. 38, No. 7-8, 1994, p. 456. doi:10.1007/BF02269836

[3] S. L. Larson and A. B. Strong, Technical Report, IRRP96-3, 1996.

[4] F. Matsui, D. L. Robertson and E. G. Lovering, "Determination of Hydrazine in Pharmaceuticals III: Hydralazine and Isoniazid Using GLC," Journal of Pharmaceutical Sciences, Vol. 72, No. 8, 1983, pp. 948-951. doi:10.1002/jps.2600720829

[5] P. E. Kester and N. D. Danielson, "Determination of Hydrazine and 1,1-Dimethylhydrazine as Salicyldehyde Derivates by Liquid Chromatography with Electrochemical Detection," Chromatographia, Vol. 18, No. 3, 1984, pp. 125-128. doi:10.1007/BF02258767

[6] G. W. Watt and J. D. Chrisp, "Spectrophotometric Method for Determination of Hydrazine," Analytical Chemistry, Vol. 24, No. 12, 1952, pp. 2006-2008.

doi:10.1021/ac60072a044
Table 2. Typical results of hydrazine in water samples (Water samples were collected from various located in Chhatisgarh state, India).

\begin{tabular}{ccc}
\hline S.No & Absorbance & Conc. of Hydrazine $(\mu \mathrm{g} / \mathrm{ml})$ \\
\hline S1 & 0.814 & 534.054 \\
S2 & 0.660 & 828.375 \\
S3 & 0.528 & 1035.469 \\
S4 & 0.878 & 406.524 \\
S5 & 1.542 & 67.714 \\
S6 & 0.971 & 107.534 \\
S7 & 0.918 & 113.743 \\
\hline
\end{tabular}

[7] Y. Y. Liu, J. Schemeltz and D. Hoffman, "Chemical Studies on Tobacco Smoke. Quantitative Analysis of Hydrazine in Tobacco and Cigarette Smoke," Analytical Chemistry, Vol. 46, No. 7, 1974, pp. 885-889. doi:10.1021/ac60343a046

[8] G. Neurath and W. J. Luttich, "Gas Chromatographic Separation of 5-Nitro-2-hydroxybenzal Derivatives of Unsymmetric Hydrazines," Journal of Chormatography A, Vol. 34, 1968, pp. 257-258. doi:10.1016/0021-9673(68)80047-0

[9] S. Ganesh, F. Khan, M. K. Ahmed and S. K. Pandey, "Sequential Determination of Free Acidity and Hydrazine in Presence of Hydrolysable Ions," Radiochimica Acta, Vol. 99, No. 9, 2011, pp. 587-591. doi:10.1524/ract.2011.1836

[10] S. Ganesh, F. Khan, M. K. Ahmed and S. K. Pandey, "Potentiometric Determination of Free Acidity in Presence of Hydrolysable Ions and Sequential Determination of Hydrazine," Talanta, Vo. 85, No. 2, 2011, pp. 958-963. doi:10.1016/j.talanta.2011.05.001

[11] C. Gojon and B. Dureault, "Spectrophotometric Study of the Reaction between Hydrazine and p-Dimethylaminobenzaldehyde," Journal of Nuclear Science and Technology, Vol. 33, No. 9, 1966, pp. 731-735. doi:10.3327/jnst.33.731

[12] R. Kaveeshwar and V. K. Gupta, "A New Spectrophotometric Method for the Determination of Hydrazine in Environmental Samples," Fresenius' Journal of Analytical Chemistry, Vol. 344, No. 3, 1992, pp. 114-117. doi:10.1007/BF00325125

[13] S. Amlathe and V. K. Gupta, "Spectrophotometric Determination of Trace Amounts of Hydrazine in Polluted Water," Analyst, Vol. 13, No. 9, 1988, pp. 1481-1483. doi:10.1039/an9881301481

[14] J. Manes, P. Campillos, G. Front, H. Martre and P. Prognon, "Extraction-Spectrophotometric Determination of Hydrazine with 2-Hydroxy-1-naphthaldehyde," Analyst, Vol. 112, No. 8, 1987, pp. 1183-1184. doi:10.1039/an9871201183

[15] A. Besada, "Analytical Use of Copper(II)-Neocuproine in the Spectrophotometric Determination of Hydrazines," Analytical Letters, Vol. 21, 10, 1988, pp. 1917-1925. doi:10.1080/00032718808066357

[16] O. S. Sire and J. Burno, Talanta, Vol. 47, 1979, p. 26.

[17] M. Roth and J. Rieder, "Spektrofluorometrische Bestim- 
mung von Hydrazinen und Deren Anwendungzur Quantitativen Bestimmung von Benzylhydrazin in Biologischen Proben," Analytica Chimica Acta, Vol. 27, 1962, pp. 2026. doi:10.1016/S0003-2670(00)88442-1

[18] T. Pal, D. S. Maity and A. Ganguly, "Use of a SilverGelatin Complex for the Determination of MicroAmounts of Hydrazine in Water," Analyst, Vol. 111, No. 12, 1986, pp. 1413-1415. doi:10.1039/an9861101413

[19] A. Besada, "A Simple Spectrophotometric Method for the Determination of Hydrazine Salts by Reaction with Phosphomolybdic Acid," Mikrochimica Acta, Vol. 111, No. 5-6, 1985, pp. 343-346. doi:10.1007/BF01196832

[20] R. P. V. Krishna, R. R. Samba Siva and C. Rambabu, Acta Ciencia Indica, Vol. 4, 1978, p. 13.

[21] M. George, K. S. Nagaraj and N. Balasubramanian, "Spectrophotometric Determination of Hydrazine," Talanta, Vol. 75, No. 1, 2008, pp. 27-31. doi:10.1016/j.talanta.2007.09.002

[22] A. M. Haji Shabani, S. Dadfarnia and K. Dehghan, "Indirect Spectrophotometric Determination of Trace Quantities of Hydrazine," Bulletin of the Korean Chemical Society, Vol. 25, No. 2, 2004, pp. 213-215. doi:10.5012/bkcs.2004.25.2.213

[23] J. Mendham, R. C. Denney, J. D. Barnes and M. Thomas, "Vogel's Text Book of Quantitative Chemical Analysis," 6th Edition, 2002, p. 441.

[24] S. Ganesh, F. Khan, M. K. Ahmed and S. K. Pandey, "Sequential Determination of Uranium(IV), Free Acidity and Hydrazine in a Single Aliquot," Journal of Radioanalytical and Nuclear Chemistry, Vol. 286, No. 1, 2010, pp. 33-37. doi:10.1007/s10967-010-0663-9

[25] J. Mendham, R. C. Denney, J. D. Barnes and M. Thomas, "Vogel's Text book of Quantitative Chemical Analysis," 6th Edition, 2002, p. 350. 